\title{
Fragilidade Financeira e Volatilidade dos Ciclos Econômicos no Brasil Pós-Plano Real
}

\author{
Diego Nunes Teixeira ${ }^{1}$ \\ Gisele Ferreira Tiryaki ${ }^{2}$ \\ Carlos Eduardo Iwai Drummond ${ }^{3}$
}

\section{Resumo}

Fricções financeiras comprometem a eficiência com que sistema financeiro direciona recursos para o financiamento de gastos com consumo e com investimentos em bens de capital. Imperfeições no mercado financeiro elevam a volatilidade dos ciclos de econômicos, em razão de flutuações no prêmio de financiamento externo ou de mudanças no comportamento de risco dos agentes econômicos. Este artigo investiga se um aumento da fragilidade financeira compromete a estabilidade dos ciclos econômicos no Brasil. Utilizando dados mensais entre 1996 e 2018, um indicador de fragilidade financeira foi derivado com base no modelo de fator dinâmico e a estimação de modelos de vetores autorregressivos permitiu analisar o comportamento deste indicador nos ciclos econômicos. Os resultados indicam que um aumento na fragilidade financeira compromete o desempenho macroeconômico e revelam a importância da volatilidade cambial e do componente cíclico do nível de preços para as flutuações de curto prazo na atividade econômica no Brasil.

\section{Palavras-Chave}

Ciclos econômicos. Instabilidade financeira. Fragilidade.

\author{
Abstract \\ Financial frictions undermine the efficiency with which the financial system channels resources \\ to fund consumption expenditures and fixed capital investment. Fluctuations in the external \\ finance premium or changes in the risk behavior of economic agents may lead to increased \\ business cycles volatility. This paper investigates whether greater financial fragility increases the \\ short-run fluctuations in economic activity in Brazil. Using monthly data between 1996 and \\ 1 Pesquisador - Universidade Federal da Bahia (UFB) \\ Endereço: Praça da Piedade, 06 - Salvador/BA - Brasil - CEP: 40070-010. \\ E-mail: diegont2@yahoo.com.br - ORCiD: https://orcid.org/0000-0002-9945-7902. \\ 2 Professora - Universidade Federal da Bahia (UFB) \\ Endereço: Praça da Piedade, 06 - Salvador/BA - Brasil - CEP: 40070-010. \\ E-mail: gtiryaki@ufba.br - ORCiD: https://orcid.org/0000-0002-2608-5378. \\ 3 Professor - Universidade Estadual de Santa Cruz - Campus Soane Nazaré de Andrade \\ Rodovia Jorge Amado, km 16 - Salobrinho - Brasil - CEP: 90040-000. \\ E-mail: ceidrumond@uesc.br - ORCiD: https://orcid.org/0000-0002-2865-1804. \\ Recebido: 06/12/2017. Aceite: 13/09/2019. \\ Editor Responsável: Dante Mendes Aldrighi
}

(c) (i) (\$) Esta obra está licenciada com uma Licença Creative Commons Atribuição-Não Comercial 4.0 Internacional. 
2018, this article applied a dynamic factor model to derive a financial system fragility indicator and estimated vector autoregressive models to evaluate this indicator's performance throughout the business cycles. The results indicate that greater financial fragility compromise the country's macroeconomic performance and reveal the importance of exchange-rate volatility and price level volatility to the short-run fluctuations in economic activity in Brazil.

\section{Keywords}

Business cycles. Financial instability. Fragility.

\section{JELClassification}

E32. E44. E5.

\section{Introdução}

Desenvolvimentos recentes da literatura em macroeconomia apontam para uma estreita relação entre as imperfeições no mercado de crédito e o comportamento cíclico da produção, uma conclusão que se distancia daquela indicada por Kydland e Prescott (1982) e Long e Plosser (1983), precursores da teoria dos ciclos reais de negócios. Dificuldades associadas à assimetria de informação entre demandadores e ofertantes de recursos financeiros, ao comportamento de risco das instituições financeiras, frente às pressões competitivas no mercado e aos erros sistemáticos de previsão de agentes econômicos, afetam o funcionamento do mercado financeiro e podem desencadear ou amplificar crises econômicas.

A importância do funcionamento do mercado de crédito para as flutuações na atividade econômica é comumente enfatizada pelas análises de tradição Keynesiana. Os primeiros trabalhos mais influentes procuravam mostrar como o comportamento de risco dos agentes econômicos e as imperfeições no mercado de crédito comprometiam o desempenho da economia (Minsky 1986, Bernanke et al. 1996 e 1999).

A partir da segunda metade dos anos 2000, observa-se uma mudança metodológica nos estudos sobre ciclos econômicos, que passaram a se utilizar majoritariamente de modelos de equilíbrio geral dinâmico estocástico (DSGE) com competição imperfeita e rigidez nominal e real, mas que não incorporavam explicitamente fricções financeiras (Christiano et al. 2005, 
Smets e Wouters 2007). Com a crise de 2008, reconheceu-se a dificuldade dos modelos DSGE Novo-Keynesianos e a literatura começou a se ajustar rapidamente para incorporar imperfeições financeiras ao arcabouço teórico (Gilchrist e Zakrajšek 2012, Gilchrist et al. 2014).

O presente artigo tem por objetivo analisar como a fragilidade financeira se relaciona com o comportamento cíclico da economia brasileira no período após o Plano Real. Utilizando dados mensais para o período entre 1996 e 2018, o trabalho busca verificar se choques na fragilidade financeira comprometem o desempenho econômico de curto prazo, levando a uma contração na renda.

Diversos trabalhos empíricos procuram estabelecer a relação entre fricções financeiras e os ciclos econômicos. Caballero e Krishnamurthy (2001) e Aghion et al. (2010) afirmam que os efeitos das flutuações na atividade econômica em países com maior robustez do mercado financeiro são menores, particularmente em períodos de menor liquidez do mercado internacional. Dabla-Norris e Srivisal (2013), por sua vez, mostram que países com maior robustez do setor financeiro e alta abertura comercial e financeira estão menos sujeitos aos impactos nos termos de troca e choques de preços externos, como os preços de commodities, sobre o consumo.

Trabalhos mais recentes têm discutido o papel das fricções no mercado financeiro sobre a volatilidade dos preços dos ativos e dos spreads bancários, buscando avaliar como tais fricções afetam os agregados macroeconômicos. Gilchrist e Zakrajšek (2012) e Gilchrist et al. (2014), por exemplo, afirmam que elevações na incerteza conduzem ao declínio na atividade econômica. Adicionalmente, os autores salientam que as distorções financeiras, medidas por variações no spread de crédito, apresentam impactos significativos na economia, independentemente do nível de incerteza.

Observa-se que a maioria dos trabalhos empíricos que buscam estudar a relação entre fragilidade financeira e volatilidade dos agregados macroeconômicos utiliza dados cross-country, sem levar em consideração a dinâmica de curto prazo que só é possível analisar em séries temporais com frequência mensal ou trimestral. Excetuam-se os trabalhos de Teixeira e Tiryaki (2017), Tiryaki et al. (2017) e Charles et al. (2018), que possuem objetivos similares ao presente trabalho, mas adotam estratégias empíricas e bases de dados diferenciados e obtêm conclusões distintas. 
Tiryaki et al. (2017) utilizam um modelo de vetores autorregressivos (VAR) para estabelecer a relação entre inadimplência, crédito e os ciclos econômicos no Brasil. Os autores analisam apenas o desempenho cíclico das variáveis de concessão de crédito, inadimplência e taxas de juros, sem levar em consideração outros determinantes da fragilidade financeira e sem buscar derivar formalmente um índice de fragilidade financeira. Adicionalmente, aqueles autores concluem que choques na produção elevam a fragilidade financeira.

Os resultados do presente trabalho obtidos com a estimação de modelos VAR indicam uma reversão da causalidade apontada por Tiryaki et al. (2017). Aqui, os resultados sugerem que choques exógenos na fragilidade financeira comprometem o desempenho macroeconômico. Essa distinção é importante para identificar a análise teórica da relação entre fragilidade financeira e volatilidade cíclica da economia que melhor adere à realidade empírica. Adicionalmente, a análise empírica indica que choques cambiais elevam a fragilidade financeira e comprometem o desempenho macroeconômico.

O presente artigo também inova ao construir um indicador de fragilidade financeira para o Brasil utilizando o modelo de fator dinâmico. Esta metodologia permite incorporar um conjunto maior de variáveis, como medidas de liquidez, de prêmio de risco e de fragilidade no mercado de capitais, ausentes no trabalho de Tiryaki et al. (2017). O modelo de fator dinâmico também foi empregado por Charles et al. (2018) para derivar um indicador de incerteza com dados da economia americana, que foi utilizado posteriormente na estimação de modelos VAR. Estes autores concluíram que a incerteza contribui de maneira significativa para a volatilidade macroeconômica.

Além desta introdução, o artigo é composto por mais quatro seções. A segunda seção apresenta uma breve revisão da literatura que enfatiza a relação entre instabilidade financeira e os ciclos econômicos. Na terceira seção são descritos os dados e as variáveis utilizadas no trabalho, além do modelo de fator dinâmico utilizado para derivar o índice de fragilidade financeira. A quarta seção apresenta a metodologia econométrica, enquanto a quinta seção discute os resultados obtidos na análise empírica. A última seção apresenta as considerações finais. 


\section{Instabilidade Financeira e Ciclos Econômicos}

O sistema financeiro tem papel central na promoção do desenvolvimento e na estabilidade macroeconômica de um país. No longo prazo, economias com sistemas financeiros melhor estruturados e capacitados para selecionar e monitorar projetos de investimento apresentam maior crescimento econômico e reduções mais rápidas nos níveis de pobreza (King e Levine 1993, e Levine 2005). No curto prazo, no entanto, a relação entre desenvolvimento financeiro e estabilidade macroeconômica é considerada ambígua, visto que inovações financeiras presentes no processo de desenvolvimento financeiro podem gerar instabilidade e amplificar a volatilidade da atividade econômica, particularmente em mercados financeiros mais competitivos (Gennaioli et al., 2012; Marques-Ibanez et al., 2014 e Beck et al., 2016).

$\mathrm{Na}$ análise clássica, particularmente aquela associada à teoria dos ciclos reais de negócios, o sistema financeiro não é relevante para as flutuações econômicas de curto prazo, que são consideradas respostas ótimas da economia aos choques de produtividade ou de gastos do governo (Kydland e Prescott 1982, Long e Plosser 1983). Já para teóricos de tradição Keynesiana, as fricções financeiras contribuem de maneira significativa para a volatilidade dos ciclos econômicos. Essa seção apresenta a discussão teórica e empírica sobre a importância das flutuações na aversão ao risco dos agentes econômicos, da assimetria de informação e da incerteza no mercado financeiro para as flutuações econômicas de curto prazo.

A instabilidade do sistema financeiro pode ser considerada inerente às economias de mercado, assim como as flutuações nos agregados macroeconômicos (Minsky 1986, Fazzari et al. 2008, Ryoo 2010, Borio e Zhu 2012, Azariadis 2018). Neste caso, a instabilidade financeira é resultado do comportamento de risco dos agentes econômicos. Em períodos de estabilidade, quando as expectativas sobre o desempenho futuro da economia são favoráveis, os agentes reduzem sua aversão ao risco e tornam-se mais alavancados. O otimismo generalizado e pressões competitivas levam as instituições financeiras a expandirem a oferta de crédito e a facilitarem as condições de financiamento. Quanto maior a quantidade de agentes econômicos com reduzida aversão ao risco e com expectativas exageradamente otimistas sobre o desempenho econômico, maior será a probabilidade de fragilização do sistema financeiro. 
Portanto, as flutuações de curto prazo da atividade econômica são interpretadas como resultado de modificações na predisposição ao risco dos agentes econômicos, em razão de mudanças nas crenças e no estado de ânimo. O processo de expansão da atividade econômica prossegue enquanto as instituições financeiras estiverem dispostas a validar essas posições financeiras mais arriscadas, mas essa expansão no crédito cessará tão logo o grau de fragilidade financeira mostre-se insustentável.

A literatura sobre os mecanismos de transmissão de política monetária via canais do crédito também enfatiza a importância do sistema financeiro para as flutuações de curto prazo da atividade econômica (Stiglitz 1987, Gertler 1988, Bernanke e Lown 1991, Bernanke et al. 1996, 1999). Imperfeições no mercado financeiro associadas à assimetria de informações entre credores e devedores fazem com que o impacto das intervenções do banco central sobre a atividade econômica seja amplificado, o chamado "acelerador financeiro". Períodos de expansão na atividade econômica, quando se observa a elevação na riqueza, seriam acompanhados por problemas menos severos de assimetria de informação e, consequentemente, maior robustez financeira.

A relação inversa entre o risco de financiar e o nível de riqueza dos mutuários é resultado da percepção do sistema financeiro de que a elevação no patrimônio líquido é um bom indicador da saúde financeira dos agentes econômicos. Assim, diferente do canal do risco explorado por Minsky (1986), Fazzari et al. (2008), Ryoo (2010), Borio e Zhu (2012), e Azariadis (2018), expansões na atividade econômica permitem a redução da razão endividamento-capital próprio, reduzindo o impacto de problemas associados à assimetria de informação e garantindo uma maior robustez do sistema financeiro.

O efeito do acelerador financeiro decorre do caráter anticíclico do prêmio de risco associado à assimetria de informação: elevações no patrimônio líquido dos agentes econômicos observadas durante as expansões da atividade econômica sinalizam a redução no risco associado aos problemas de seleção adversa e risco moral, estimulando a concessão de crédito (Gertler 1988, Bernanke et al 1996, 1999, Gilchrist e Zakrajšek 2012). O spread bancário é, portanto, um importante indicador dos custos de agenciamento das instituições financeiras, influenciando a dinâmica do mercado de crédito da economia. $\mathrm{O}$ aumento do prêmio de risco sinaliza uma deterio- 
ração das condições do sistema financeiro, reduzindo o fluxo de recursos financeiros e retraindo os investimentos e consumo das famílias.

Stiglitz (1987) afirma que a percepção de qualidade do mutuário estabelecida pela instituição financeira depende do comprometimento de recursos próprios do mutuário com o projeto de investimento. Elevações nas taxas de juros resultantes de intervenções de política monetária aumentam a probabilidade de default, pois somente projetos de maior risco são viabilizados, reduzindo a qualidade dos demandantes de crédito. Desse modo, as instituições financeiras podem simplesmente racionar crédito, ao invés de adotar taxa de juros de mercado mais elevadas.

De maneira semelhante, Mishkin $(1998,2000)$ afirma que a instabilidade financeira decorre de problemas no funcionamento do sistema financeiro que comprometem os fluxos de informação e a canalização de recursos para projetos de investimento produtivo. Este autor indica que quatro fatores, simultaneamente ou não, podem aumentar a probabilidade de surgimento de instabilidades no sistema financeiro:

(i) A deterioração dos balanços patrimoniais das instituições financeiras, normalmente fruto do gerenciamento precário de um processo de liberalização financeira;

(ii) A deterioração dos balanços patrimoniais das firmas, devido à redução nos preços das ações, no nível preço esperado ou uma depreciação cambial;

(iii) Aumentos das taxas de juros, em função de um aquecimento na demanda por crédito ou como resultado de intervenções de política monetária; e,

(iv) Aumento da incerteza, comumente associada à fragilização de instituições financeiras de porte ou pânico bancário.

Para o autor, todos esses fatores fazem com que os problemas de assimetria de informação se agravem, tornando mais difícil para as instituições financeiras selecionar projetos de investimento robustos e monitorar o uso de recursos. Como consequência, o racionamento de crédito se eleva, assim como o prêmio de risco associado às taxas de juros, o que impacta negativamente sobre o investimento e o desempenho da economia. 
Mais recentemente, Gilchrist, Sim e Zakrajšek (2014) mostraram que mudanças na incerteza conduzem ao declínio na atividade real da economia, principalmente pela prolongada queda dos investimentos. Ademais, afirmam que as distorções financeiras provocadas pelos choques de spread apresentam impactos significativos na economia, independentemente do nível de incerteza.

\subsection{Evidência Empírica}

O aumento da volatilidade dos ciclos econômicos tem efeitos negativos sobre o crescimento econômico (ver Ramey e Ramey 1994). A irreversibilidade do investimento, por exemplo, pode fazer com que elevações na volatilidade dos ciclos econômicos prejudiquem o desempenho de longo prazo de uma economia. Além disso, se os ciclos econômicos são associados ao aumento na volatilidade da taxa de inflação, pode haver um desestímulo ao investimento e, consequentemente, menor crescimento econômico.

Os primeiros trabalhos empíricos sobre o sistema financeiro enfatizavam a conexão entre desenvolvimento financeiro e crescimento econômico (King e Levine 1993, Levine 2005; Čihák et al. 2013). De acordo com esses autores, o desenvolvimento do sistema financeiro permite que um maior volume de crédito seja direcionado para o investimento, estimulando o crescimento econômico.

Posteriormente, outros trabalhos indicaram que o desenvolvimento do sistema financeiro também tendia a minimizar a volatilidade dos ciclos econômicos, provavelmente em função da maior eficiência operacional das instituições financeiras (ver, por exemplo, Denizer et al. 2002, Silva 2002, Dabla-Norris e Srivisal 2013). No entanto, a relação entre desenvolvimento financeiro e volatilidade cíclica da economia é ambígua, visto que inovações financeiras, normalmente associadas às pressões competitivas, podem vir acompanhadas de comportamentos mais arriscados por parte dos agentes do mercado financeiro, gerando instabilidade (Gennaioli et al. 2012, Marques-Ibanez et al. 2014, Beck et al. 2016).

Easterly et al (2000) mostram que períodos de expansão no crédito, principalmente em países com razão de oferta de crédito ao setor privado maior que $100 \%$ do $\mathrm{PIB}$, estão associados à uma maior volatilidade do 
produto agregado. Já Loayza e Raddatz (2007) e Dabla-Norris e Srivisal (2013), por sua vez, encontram evidências de que os choques externos são absorvidos de maneira mais suave em economias com mercados financeiros mais desenvolvidos. Por fim, Popov (2011) encontra evidências de uma relação positiva entre crescimento e liberalização financeira, embora seus resultados também indiquem que maior liberalização financeira pode potencializar os períodos de crises.

Mais recentemente, vários trabalhos têm se dedicado a derivar índices de incerteza econômica e de fragilidade financeira, verificando sua relação com o desempenho macroeconômico (Kliesen et al. 2012, Jurado et al. 2015, Baker et al. 2016, Charles et al. 2018). Charles et al. (2018), em particular, adotam estratégia semelhante ao presente trabalho, utilizando o modelo de fator dinâmico para derivar uma medida única de incerteza e verificar sua relação com variáveis macroeconômicas no curto prazo por meio de vetores autorregressivos. Esses trabalhos foram em sua maior parte desenvolvidos com dados da economia americana e desconhece-se iniciativa semelhante de derivação de índice de fragilidade com o uso de fator dinâmico para o Brasil.

\section{Dados e Variáveis}

O Brasil apresenta um elevado nível de concentração bancária, com os cinco maiores bancos detendo cerca de $80 \%$ dos ativos bancários (World Bank, 2017). Essa baixa competitividade tem aparentemente promovido uma menor exposição ao risco por parte das instituições financeiras, que vêm apresentando uma tendência declinante no percentual de empréstimos não liquidados e no indicador Z-Score ao longo dos últimos dez anos (World Bank 2017). ${ }^{1}$

Outro elemento que tem sinalizado a redução da exposição ao risco do sistema financeiro brasileiro é o comportamento do prêmio de risco. Dados do BCB (2019) indicam que houve declínio no spread das operações no mercado de crédito no Brasil no período pós-Real, com o prêmio de risco se elevando de maneira moderada mais recentemente, em razão da crise econômica.

1 O Z-Score é um indicador de solvência bancário, cujo cálculo pode ser obtido em Beck et al. (2007). 
Neste mesmo período, também se observa uma tendência de redução da volatilidade do mercado acionário brasileiro, embora se constate que os preços das ações se distanciam de maneira substancial do seu valor fundamental em períodos de instabilidade econômica. De acordo com BCB (2019), foram observadas maiores discrepâncias entre as taxas de crescimento do valor das ações e da atividade econômica entre 1997 e 2002, período marcado por grande instabilidade nos mercados globais. O desvio médio deste diferencial só voltou a ser mais representativo em 2008 e a partir de 2016, refletindo novamente momentos de grande instabilidade macroeconômica.

Uma avaliação do comportamento do mercado financeiro ao longo dos ciclos econômicos requer a utilização de dados com frequência mensal ou trimestral. Neste sentido, o presente estudo utilizou dados mensais da economia brasileira entre janeiro de 1996 e julho de 2018, obtidos em maior parte junto ao BCB (2019). ${ }^{2}$ Esta seção dedica-se, a princípio, a apresentar as variáveis utilizadas na construção do indicador de fragilidade financeira e o modelo de fator dinâmico utilizado para a derivação deste índice, e, posteriormente, a descrever as variáveis utilizadas na análise econométrica.

\subsection{Indicador de Fragilidade Financeira}

$\mathrm{O}$ indicador de fragilidade financeira incorporou nove variáveis que refletem o desempenho do sistema financeiro. As três primeiras variáveis são medidas de desenvolvimento do sistema financeiro e as demais capturam flutuações no prêmio de risco e a presença de bolhas no mercado de capitais. ${ }^{3}$

Sistemas financeiros desenvolvidos possuem melhor capacidade de lidar com os problemas de assimetria de informação e, por consequência, de selecionar projetos menos arriscados, contribuindo para a promoção da estabilidade macroeconômica de curto prazo. Baseando-se em King e Levine (1993) e Levine (2005), três variáveis de desenvolvimento do sistema fi-

2 Dados obtidos de fontes alternativas são sinalizados ao longo da seção.

3 Outras variáveis são passíveis de inclusão na análise, como, por exemplo, a volatilidade das variáveis já selecionadas para capturar choques de segundo momento. No entanto, a elevação no número de variáveis dificulta a convergência de modelos de fator dinâmico baseados no filtro de Kalman, particularmente quando o número de observações é restrito, sem elevar a precisão das estimativas (Poncela e Ruiz 2016). 
nanceiro foram utilizadas, com a primeira representando o grau de liquidez do sistema financeiro e as outras duas representando proxies para robustez do mercado de crédito:

- MlPIB: razão entre o saldo mensal dos meios de pagamento (Ml), ajustado sazonalmente, e o PIB mensal acumulado nos últimos doze meses;

- PRIVY: razão entre crédito destinado a setor privado e PIB mensal acumulado nos últimos doze meses;

- CREDPRIV: razão entre crédito destinado a setor privado e crédito total.

As outras seis variáveis utilizadas na construção do índice buscaram mensurar as flutuações nos custos de agenciamento e no grau de exposição ao risco do sistema financeiro. A literatura sobre assimetria de informação aponta que, em momentos de contração na atividade econômica, reduções no patrimônio dos agentes econômicos elevam o prêmio com o financiamento utilizando recursos de terceiros, refletindo maiores custos de agenciamento. Desse modo, supõe-se que o spread de risco das taxas de juros aumente em períodos de maior instabilidade financeira e, consequentemente, maior risco de default.

Adicionalmente, instituições financeiras que se expõem exageradamente ao risco na fase de expansão do ciclo econômico apresentam uma deterioração em suas demonstrações financeiras, apresentando perdas com liquidação de créditos duvidosos. A consequente contração no crédito também é acompanhada de elevação no spread bancário.

Desta forma, as seguintes variáveis foram utilizadas como indicadores do prêmio risco com financiamentos, do grau de exposição ao risco das instituições financeiras e de bolhas no mercado de capitais: ${ }^{4}$

- PFSELIC: diferença entre a taxa média de juros de crédito pessoal - pessoa física e a taxa SELIC;

- PJSELIC: diferença entre a taxa média de juros das operações crédito com pessoa jurídica (duplicatas e recebíveis) e a SELIC;

\footnotetext{
${ }^{4}$ Todas as variáveis de prêmio de risco foram medidas em valores percentuais no mês, anualizados.
} 
- CHQSELIC: diferença entre a taxa média de juros de cheque especial e a SELIC;

- PROVPUB: razão entre a provisão para créditos de liquidação duvidosa e o total do crédito concedido pelas instituições financeiras públicas do Sistema Financeiro Nacional;

- PROVPRIV: razão entre a provisão para créditos de liquidação duvidosa e o total do crédito concedido pelas instituições financeiras privadas do Sistema Financeiro Nacional;

- BOVPIB: a razão entre o valor mensal real das empresas listadas na BOVESPA e o PIB real. Conhecido como o "índice de Buffett", é comumente utilizado para avaliar se o mercado de capitais está sobrevalorizado (Chang e Pak 2018, Pysarenko et al. 2019).

Como grande parte dos investimentos de longo prazo no Brasil são financiados com taxas de juros subsidiadas pelo Banco Nacional de Desenvolvimento Econômico e Social (BNDES), evitou-se incluir no modelo as taxas de juros de longo prazo, que poderiam refletir de maneira mais apropriada o custo de oportunidade de projetos de investimento e do consumo de bens duráveis. Não obstante, considerando que o comportamento da estrutura a termo das taxas de juros indica uma elevada correlação entre as taxas de juros de curto e longo prazo, os problemas decorrentes da ausência desta variável não devem ser relevantes.

\subsubsection{Modelo de Fator Dinâmico}

A análise de fatores permite identificar fatores latentes que determinam um comportamento comum entre variáveis. Deriva-se um número menor de componentes não correlacionados que reflete o comportamento das variáveis originais. Ao utilizar a análise de fatores para derivar o indicador de fragilidade financeira, assume-se que a fragilidade pode ser resultado do comportamento de risco dos agentes e do agravamento da assimetria de informação decorrente de choques exógenos (e.g. iniciativas de política monetária, choques em preços de commodities e inovações tecnológicas).

A análise de fatores multivariada não pode ser utilizada se os fatores seguem um processo que se modifica ao longo do tempo. Logo, no caso de 
séries temporais, em que existe a presença de componentes autorregressivos, sugere-se o uso do modelo de fator dinâmico (ver Geweke 1977, e Stock e Watson 2011).

A premissa deste modelo é que o movimento sincronizado de um vetor de séries temporais com elevada dimensão $\left(\mathrm{X}_{\mathrm{t}}\right)$ é gerado por um conjunto de poucos fatores latentes $\left(\mathrm{f}_{\mathrm{t}}\right)$ e um vetor de erros idiossincráticos com média zero $\left(e_{t}\right)$. Presume-se, também, que o vetor de fatores latentes segue um processo de séries temporais, normalmente descrito por um vetor autorregressivo.

Stock e Watson (2011) definem:

$$
\begin{aligned}
& X_{t}=\lambda(L) f_{t}+e_{t} \\
& f_{t}=\psi(L) f_{t-1}+\eta_{t}
\end{aligned}
$$

onde $\mathrm{N}$ é o número de séries temporais em $\mathrm{X}_{\mathrm{t}}$ incluídas na análise, com $\mathrm{X}_{\mathrm{t}}$ e $e_{t}$ possuindo dimensão $\mathrm{Nxl}$ e $t$ representando a unidade temporal. Existe um número q de fatores dinâmicos que compõe a matriz $f$, de forma que $\mathrm{f}_{\mathrm{t}}$ e $\eta_{t}$ possuem dimensão qxl e q possui uma dimensão bem inferior a $\mathrm{N}$. Assume-se que o componente idiossincrático, $e_{t}$, não é correlacionado com as inovações dos fatores, $\eta_{t}$. O operador de defasagem é representado por L e as matrizes polinomiais defasadas $\lambda(L)$ e $\psi(L)$ possuem dimensões $\mathrm{N}$ x q e q x q, respectivamente. O polinomial defasado $\lambda_{i}(L)$ é chamado de carga do fator dinâmico da variável $\mathrm{X}_{\mathrm{it}}, \operatorname{com} \lambda_{i}(L) f_{t}$ indicando a fração de $\mathrm{X}_{\mathrm{it}}$ que é explicada pelos componentes comuns.

Os parâmetros são estimados utilizando máxima verossimilhança com base em um filtro de Kalman. Foi possível utilizar esta estratégia porque o número de séries temporais é restrito em relação ao número de observações. Assim como em Quah e Sargent (1993) e Aruoba et al. (2009), um fator dinâmico único é estimado no presente trabalho, pressupondo-se que segue um processo autoregressivo de segunda ordem.

Em uma etapa preliminar à estimação do fator dinâmico, é preciso verificar se as variáveis originais são estacionárias. Para isso, utilizou-se dos seguintes testes de raiz unitária, comumente empregados na literatura: Augmented Dickey-Fuller (ADF), Kwiatkowski-Phillips-Schimdt-Shin (KPSS) e Dickey-Fuller GLS (DF-GLS). Os resultados destes testes estão 
apresentados no Apêndice 1 e indicam que todas as variáveis são estacionárias em primeiras diferenças.

A Tabela 1 apresenta os resultados da estimação do modelo. $O$ fator latente é persistente, tendo em vista que a primeira e segunda defasagens do fator são importantes na sua determinação (significância estatística de 10\% e $1 \%$, respectivamente). O fator também é um preditor significativo de maior parte das variáveis incluídas. As variáveis relacionadas aos custos de agenciamento e ao grau de exposição ao risco do sistema financeiro relacionam-se positivamente com o indicador de fragilidade financeira, enquanto que PRIVY, uma das medidas de desenvolvimento financeiro, mostrou-se negativamente relacionada com a medida de fragilidade financeira. As variáveis PROVPUB, BOVPIB, CREDPRIV e MIPIB não apresentam relação estatisticamente significante com o fator latente.

Tabela 1 - Resultados do Modelo de Fatores Dinâmico

\begin{tabular}{lcccccc}
\hline & Coeficiente & Desvio-padrão & $\mathbf{z}$ & $\mathbf{P}>|\mathbf{z}|$ & \multicolumn{2}{c}{$[95 \%$ Interval, Conf.] } \\
\hline L1. & 0,134 & 0,081 & 1,66 & 0,097 & $-0,024$ & 0,293 \\
L2. & 0,347 & 0,119 & 2,92 & 0,003 & 0,114 & 0,580 \\
\hline D.PJSELIC & 1,154 & 0,165 & 6,99 & 0,000 & 0,831 & 1,478 \\
D.PFSELIC & 1,722 & 0,264 & 6,52 & 0,000 & 1,204 & 2,239 \\
D.BOVPIB & $-0,002$ & 0,002 & $-1,43$ & 0,152 & $-0,005$ & 0,001 \\
D.CHQSELIC & 2,075 & 0,421 & 4,93 & 0,000 & 1,251 & 2,899 \\
D.PROVPUB & 0,050 & 0,054 & 0,93 & 0,353 & $-0,055$ & 0,155 \\
D.PROVPRIV & 0,047 & 0,027 & 1,76 & 0,079 & $-0,005$ & 0,099 \\
D.CREDPRIV & 0,001 & 0,0004 & 1,51 & 0,130 & $-0,0002$ & 0,001 \\
D.PRIVY & $-0,001$ & 0,0004 & $-2,51$ & 0,012 & $-0,002$ & $-0,0002$ \\
D.M1PIB & $-0,0001$ & 0,0001 & $-1,58$ & 0,115 & $-0,0003$ & 0,00003 \\
\hline Variância: & & & & & & \\
De.PJSELIC & 4,159 & 0,437 & 9,51 & 0,000 & 3,302 & 5,016 \\
De.PFSELIC & 2,428 & 0,770 & 3,15 & 0,001 & 0,919 & 3,937 \\
De.BOVPIB & 0,001 & 0,00005 & 11,6 & 0,000 & 0,0005 & 0,001 \\
De.CHQSELIC & 35,201 & 3,288 & 10,7 & 0,000 & 28,757 & 41,646 \\
De.PROVPUB & 0,458 & 0,040 & 11,6 & 0,000 & 0,380 & 0,536 \\
De.PROVPRIV & 0,136 & 0,012 & 11,5 & 0,000 & 0,113 & 0,160 \\
De.CREDPRIV & 0,00003 & $2,71 \mathrm{E}-06$ & 11,5 & 0,000 & 0,00003 & 0,00004 \\
De.PRIVY & 0,00002 & $2,12 \mathrm{E}-06$ & 11,4 & 0,000 & 0,00002 & 0,00003 \\
De.M1PIB & $1,27 \mathrm{E}-06$ & $1,10 \mathrm{E}-07$ & 11,5 & 0,000 & 0,000001 & 0,000001 \\
\hline Fonte: & & & & &
\end{tabular}

Fonte: elaboração própria. 
Na Figura 1, ilustra-se o comportamento do indicador no período em estudo, com as áreas sombreadas indicando períodos recessivos definidos pelo CODACE (2017). Observa-se que a fragilidade financeira tende a se elevar em períodos recessivos, embora se verifique movimentos menos relevantes no indicador na última recessão iniciada em 2014.

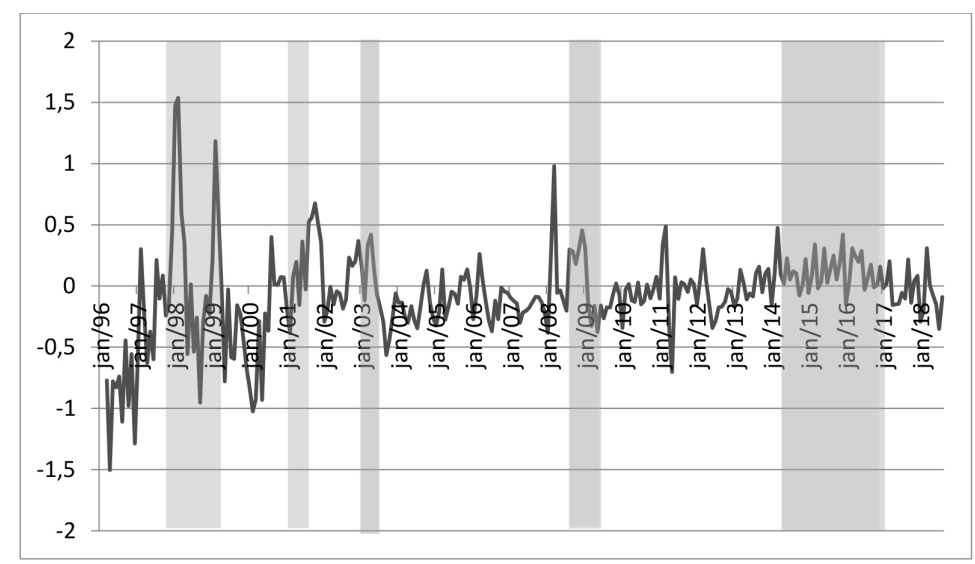

Figura 1 - Indicador de Fragilidade Financeira

Fonte: elaboração própria. Nota: áreas sombreadas representam períodos recessivos (CODACE 2017).

\subsection{Demais Variáveis Utilizadas no Modelo Econométrico}

O produto agregado real foi obtido a partir da série mensal do PIB nominal acumulado nos últimos doze meses, deflacionando-a pelo índice nacional de preços ao consumidor amplo (IPCA). A escolha pelo IPCA foi feita em virtude da indisponibilidade de dados mensais para o deflator do PIB.

Obtidos os valores reais mensais para o PIB, utilizou-se o filtro HP para obter o componente cíclico dessa variável (CYCPIB). Como os valores mensais para o PIB são estimativas, utilizaram-se também os componentes cíclicos do Índice de Atividade Econômica do Banco Central do Brasil (CYCIBC) e da produção industrial (CYCIND) como medidas alternativas de volatilidade na produção. ${ }^{5}$

5 O filtro HP empregou o valor padrão para o parâmetro de suavização com dados mensais, $\lambda=14400$. 
Também foram levadas em consideração outras variáveis identificadas pela literatura como relevantes para a volatilidade dos ciclos econômicos. Os componentes cíclicos dessas variáveis, detalhadas a seguir, também foram obtidos com os filtros HP: ${ }^{6}$

- IPCA: representa o logaritmo do índice de preços obtido a partir do IPCA. ${ }^{7}$ Esta variável é um indicador de efetividade da política monetária: elevadas taxas de inflação geram incertezas e distorções em preços relativos, contribuindo para uma maior volatilidade cíclica.

- GOV: representa um indicador de política fiscal, medido pela proporção das despesas do governo em relação ao PIB, com dados obtidos em Tesouro Nacional (2019). A política fiscal é normalmente utilizada para atenuar os ciclos econômicos, mas é comum observar, particularmente em países em desenvolvimento, um comportamento pró-cíclico dos gastos públicos, contribuindo para a volatilidade econômica (ver Alesina e Campante 2008).

- SOLOW: captura o impacto de choques exógenos de produtividade na economia (Rebelo 2005). Esses choques são mensurados com a extração do componente cíclico do resíduo de Solow. ${ }^{8}$

- CAMBIO: é uma medida da instabilidade cambial, obtida pelo desvio padrão das taxas nominais diárias de câmbio no mês, expressa em US\$/R \$. Abstraindo os regimes de câmbio fixo, que são mais suscetíveis a ataques especulativos, taxas de câmbio estáveis facilitam o planejamento dos agentes econômicos, reduzindo a volatilidade dos ciclos econômicos.

Como teste de robustez dos resultados, os componentes cíclicos também foram obtidos utilizando o filtro proposto por Hamilton (2018) e indicados pelo prefixo HAM. O uso do filtro HP pode produzir relações dinâmicas espúrias e apresentar viés de final da amostra (Hamilton 2018, Schüler 2018). Filtros alternativos de maior precisão, como os filtros do tipo band-pass de Baxter e King (1999) e Christiano e Fitzgerald (2003), levam a uma perda significativa de observações. O filtro de Hamilton produz

${ }^{6}$ O componente cíclico é indicado pelo prefixo CYC associado ao nome da variável.

7 Obteve-se a inflação em cada mês acumulada nos últimos doze meses e transformou-se a variável em índice de preços utilizado como base Janeiro - 1995.

8 O resíduo é obtido pela diferença entre a variação no logaritmo da produção em dois períodos consecutivos e o produto de $(1-\alpha)$ pela variação no logaritmo do emprego. Este trabalho segue o procedimento adotado por Backus et al (1992) e Karras e Song (1996), estabelecendo $\alpha=0,36$. Os dados de emprego foram obtidos em IBGE (2017). 
melhores estimativas no final da amostra do que o filtro HP e leva à perda de um menor número de observações que os filtros band-pass, mas apresenta a desvantagem de enfatizar flutuações com duração mais longas que um ciclo econômico típico e de ignorar flutuações com duração inferior a dois anos (Schüler 2018).

\section{Metodologia Econométrica}

O modelo VAR é estruturado como um sistema de equações em que cada variável endógena é função dos seus valores defasados, dos valores atuais e defasados das demais variáveis endógenas e exógenas que compõem o sistema e do termo de erro (Lütkepohl 2005, Pesaran e Shin 1998). O modelo VAR é definido pela expressão:

$$
x_{t}=\sum_{i=1}^{p} \Phi_{i} x_{t-i}+\Psi w_{t}+\varepsilon_{t}, \quad t=1,2, \ldots, T,
$$

onde $x_{t}=\left(x_{1 t}, x_{2 t}, \ldots, x_{m t}\right)^{\prime}$ é um vetor $\mathrm{m} \times 1$ de variáveis endógenas; p é o número de defasagens; $w_{t}$ é um vetor q x 1 de variáveis exógenas e/ou determinísticas; e $\Phi_{i}$ e $\Psi$ são matrizes de coeficientes com dimensões $\mathrm{m} \times \mathrm{m}$ e $\mathrm{m} \times \mathrm{q}$, respectivamente.

De acordo com Pesaran e Shin (1998), o modelo VAR é fundamentado em três suposições:

(i) $E\left(\varepsilon_{t}\right)=0, E\left(\varepsilon_{t} \varepsilon_{t}^{\prime}\right)=\sum$ para todo $t$, onde $\sum=\left(\sigma_{i j}, i, j=1,2, \ldots m\right)$ é uma matriz positiva definida com dimensão $\mathrm{m} \mathrm{x} \mathrm{m;} E\left(\varepsilon_{t} \varepsilon_{t}^{\prime}\right)=0$ para todo $t=t^{\prime}$ e $E\left(\varepsilon_{t} \mid w_{t}\right)=0$;

(ii) Todas as raízes de $\left|I_{m}-\sum_{i=1}^{p} \Phi_{i} z^{i}\right|=0$ estão fora do círculo unitário;

(iii) $x_{t-1}, x_{t-2}, \ldots, x_{t-p} e w_{t}$ não são colineares perfeitos.

A primeira condição indica que os resíduos não devem ser correlacionados, a segunda condição garante que haja estabilidade dinâmica e a terceira condição requer a ausência de colinearidade perfeita entre as variáveis do 
sistema. Atendidas essas condições, $x_{t}$ é covariância-estacionária e pode ser reescrita como média móvel infinita:

$$
x_{t}=\sum_{i=0}^{\infty} A_{i} \varepsilon_{t}+\sum_{i=0}^{\infty} G_{i} w_{t-i}, \quad t=1,2, \ldots, T,
$$

onde $A_{i}$ é a matriz de coeficientes com dimensão m x m obtida através das seguintes relações recursivas:

$A_{i}=\Phi_{1} \mathrm{~A}_{i-1}+\Phi_{2} \mathrm{~A}_{i-2}+\cdots+\Phi_{p} \mathrm{~A}_{i-p}, i=1,2, \ldots$ com $A_{0}=I_{m}$ e $A_{i}=0$ e para i $<0$ e $G_{i}=A_{i} \psi$.

Como o objetivo do presente trabalho é estimar relações de curto prazo entre as variáveis, não é indicado o uso de modelo de vetor de correção de erros, já que este pressupõe a existência de uma relação de equilíbrio de longo prazo. Os procedimentos de extração do componente de baixa frequência com o uso dos filtros e a estimação do modelo de fator dinâmico geram séries estacionárias, o que garante que os modelos VAR estimados sejam estáveis. Ademais, o uso de modelos VAR é a estratégia indicada quando não é possível identificar a verdadeira forma estrutural e pretende-se gerar previsões da trajetória futura das variáveis endógenas, que é a proposta deste artigo. ${ }^{9}$

Foram estimados cinco modelos VAR distintos:

$x=\left[\begin{array}{lllllll}\text { CYCPIB } & \text { CYCIPCA } & \text { CYCCAMBIO } & \text { CYCSOLOW } & \text { CYCGOV } & \text { FATOR }\end{array}\right]$
$x=\left[\begin{array}{lllllll}\text { CYCIBC } & \text { CYCIPCA } & \text { CYCCAMBIO } & \text { CYCSOLOW } & \text { CYCGOV } & \text { FATOR }\end{array}\right]$
$x=\left[\begin{array}{lllllll}\text { CYCIND } & \text { CYCIPCA } & \text { CYCCAMBIO } & \text { CYCSOLOW } & \text { CYCGOV } & \text { FATOR }\end{array}\right]$
$x=\left[\begin{array}{lllllll}\text { HAMPIB } & \text { HAMIPCA } & \text { HAMCAMBIO } & \text { HAMSOLOW } & \text { CYCGOV } & \text { FATOR }\end{array}\right]$
$x=\left[\begin{array}{llllll}\text { HAMIND } & \text { HAMIPCA } & \text { HAMCAMBIO } & \text { HAMSOLOW } & \text { CYCGOV } & \text { FATOR }\end{array}\right]$

9 Se o objetivo do trabalho fosse identificar uma relação de causalidade entre fragilidade financeira e as flutuações econômicas, seria apropriado estimar um modelo de VAR estrutural. Neste caso, seria preciso definir uma estratégia de identificação para os choques na fragilidade financeira e caracterizar o comportamento das variáveis relevantes pós-intervenção. 
Não foi possível utilizar o filtro de Hamilton com as variáveis IBC e GOV em razão do número limitado de observações temporais e de problemas de singularidade, respectivamente. Assim, nos modelos 4 e 5, manteve-se CYCGOV como indicador de política fiscal.

O número de defasagens, p, é o menor número que garante ausência de autocorrelação dos resíduos, verificada por meio de testes LM. A Tabela 2 apresenta os testes usuais de número de defasagens, baseados nos critérios de Erro Final de Previsão (FPE), Akaike (AIC), Schwarz (SC) e HannanQuinn (HQ). Nos modelos 1 e 2, os testes indicaram duas defasagens; no modelo 3, duas ou quatro defasagens; e nos modelos 4 e 5 , duas ou doze defasagens. Já os testes LM revelaram a ausência de correlação de resíduos quando os modelos 1, 2 e 4 são estimados com duas defasagens e quando os modelos 3 e 5 são estimados com quatro defasagens (ver Tabela 3).

A análise dos efeitos de choques em uma variável sobre as demais variáveis endógenas é realizada via estimação de funções de resposta a impulso e análise de decomposição da variância. Funções de resposta a impulso medem o efeito de choques em um determinado período no tempo sobre os valores futuros esperados de variáveis em um sistema dinâmico. Já a análise de decomposição da variância contabiliza o quanto da variância de uma variável pode ser atribuído a cada variável endógena.

Os resultados das funções de resposta a impulso e da decomposição de variância são sensíveis ao ordenamento das variáveis. Duas condutas foram adotadas para verificar a robustez dos resultados. A primeira estratégia utilizou testes de exogeneidade em bloco, que indica a ordem estatisticamente consistente das variáveis no modelo e permite estimar funções de resposta a impulso e decomposição de variância com base na decomposição de Cholesky (Lütkepohl 2005). A variável mais exógena, aquela com o menor valor da estatística chi-quadrado estimada, deve ser listada primeiro. Em seguida, são listadas as variáveis com grau crescente de endogeneidade. A Tabela 4 apresenta os resultados dos testes de exogeneidade em bloco e indicam o ordenamento apropriado para cada modelo. 
Tabela 2 - Testes de Número de Defasagens

\begin{tabular}{|c|c|c|c|c|c|c|c|c|c|}
\hline \multicolumn{5}{|c|}{ Modelo 1} & \multicolumn{5}{|c|}{ Modelo 2} \\
\hline & FPE & $\mathrm{AIC}$ & SC & $\mathrm{HQ}$ & & FPE & AIC & SC & $\mathrm{HQ}$ \\
\hline 0 & $5,900 \mathrm{E}-22$ & $-31,854$ & $-31,766$ & $-31,818$ & 0 & $3,25 E-22$ & $-32,452$ & $-32,344$ & $-32,408$ \\
\hline 1 & $4,530 \mathrm{E}-26$ & $-41,328$ & $-40,710$ & $-41,079$ & 1 & 2,11E-25 & $-39,792$ & $-39,033$ & $-39,484$ \\
\hline 2 & $1,89 e-26^{*}$ & $-42,202^{*}$ & $-41,054^{*}$ & $41,739^{\star}$ & 2 & $1,05 e-25$ & $-40,494^{*}$ & $-39,084^{*}$ & $-39,922^{*}$ \\
\hline 3 & $2,140 \mathrm{E}-26$ & $-42,080$ & $-40,402$ & $-41,404$ & 3 & $1,15 \mathrm{E}-25$ & $-40,406$ & $-38,344$ & $-39,570$ \\
\hline 4 & $2,070 \mathrm{E}-26$ & $-42,118$ & $-39,910$ & $-41,228$ & 4 & $1,14 \mathrm{E}-25$ & $-40,416$ & $-37,703$ & $-39,316$ \\
\hline 5 & $2,210 \mathrm{E}-26$ & $-42,056$ & $-39,318$ & $-40,952$ & 5 & $1,32 \mathrm{E}-25$ & $-40,286$ & $-36,922$ & $-38,921$ \\
\hline \multicolumn{5}{|c|}{ Modelo 4} & \multicolumn{5}{|c|}{ Modelo 5} \\
\hline & FPE & $\mathrm{AlC}$ & SC & $\mathrm{HQ}$ & & FPE & AIC & SC & $\mathrm{HQ}$ \\
\hline 0 & $9,16 \mathrm{E}-20$ & $-26,810$ & $-26,722$ & $-26,774$ & 0 & $3,54 \mathrm{E}-19$ & $-25,457$ & $-25,369$ & $-25,421$ \\
\hline 1 & $1,95 \mathrm{E}-24$ & $-37,565$ & $-36,947$ & $-37,316$ & 1 & 1,58E-22 & $-33,171$ & $-32,551$ & $-32,921$ \\
\hline 2 & $7,92 \mathrm{E}-25$ & $-38,468$ & $-37,320^{*}$ & $-38,005$ & 2 & $6,59 e-23$ & $-34,048$ & $-32,896^{*}$ & $-33,584^{*}$ \\
\hline 3 & 8,29E-25 & $-38,425$ & $-36,747$ & $-37,748$ & 3 & 7,38E-23 & $\mid-33,935$ & $-32,252$ & $-33,257$ \\
\hline 4 & $8,59 \mathrm{E}-25$ & $-38,392$ & $-36,183$ & $-37,501$ & 4 & 7,22E-23 & $-33,961$ & $-31,746$ & $-33,067$ \\
\hline 5 & $9,33 \mathrm{E}-25$ & $-38,314$ & $-35,576$ & $-37,210$ & 5 & $8,58 \mathrm{E}-23$ & $-33,793$ & $-31,046$ & $-32,685$ \\
\hline 6 & $1,00 \mathrm{E}-24$ & $-38,248$ & $-34,980$ & $-36,931$ & 6 & $9,36 \mathrm{E}-23$ & $-33,712$ & $-30,434$ & $-32,390$ \\
\hline 7 & $1,03 \mathrm{E}-24$ & $-38,228$ & $-34,430$ & $-36,697$ & 7 & $1,02 E-22$ & $-33,630$ & $-29,821$ & $-32,094$ \\
\hline 8 & $6,73 \mathrm{E}-25$ & $-38,668$ & $-34,339$ & $-36,923$ & 8 & $7,66 \mathrm{E}-23$ & $-33,934$ & $-29,593$ & $-32,184$ \\
\hline 9 & $6,59 \mathrm{E}-25$ & $-38,706$ & $-33,847$ & $-36,747$ & 9 & $7,35 \mathrm{E}-23$ & $-33,992$ & $-29,119$ & $-32,027$ \\
\hline 10 & $6,36 \mathrm{E}-25$ & $-38,760$ & $-33,372$ & $-36,588$ & 10 & 7,98E-23 & $-33,930$ & $-28,525$ & $-31,751$ \\
\hline 11 & $6,85 \mathrm{E}-25$ & $-38,711$ & $-32,793$ & $-36,325$ & 11 & $8,73 \mathrm{E}-23$ & $\mid-33,864$ & $-27,928$ & $-31,471$ \\
\hline 12 & $5,76 \mathrm{e}-25^{\star}$ & $-38,913^{*}$ & $-32,465$ & $-36,314$ & 12 & $7,42 E-23$ & $-34,057$ & $-27,590$ & $-31,450$ \\
\hline
\end{tabular}

Fonte: elaboração própria. Notas: Erro Final de Previsão (FPE), Akaike (AIC), Schwarz (SC) e Hannan-Quinn (HQ). Todos os testes foram conduzidos com 12 defasagens, mas somente são apresentados os resultados relevantes para a análise. 
Tabela 3 - Teste LM de Autocorrelação dos Resíduos

\begin{tabular}{|c|c|c|c|c|c|c|c|c|c|c|c|c|c|c|}
\hline \multicolumn{3}{|c|}{ Modelo 1} & \multicolumn{3}{|c|}{ Modelo 2} & \multicolumn{3}{|c|}{ Modelo 3} & \multicolumn{3}{|c|}{ Modelo 4} & \multicolumn{3}{|c|}{ Modelo 5} \\
\hline \multicolumn{3}{|c|}{$p=2$} & \multicolumn{3}{|c|}{$p=2$} & \multicolumn{3}{|c|}{$p=4$} & \multicolumn{3}{|c|}{$p=2$} & \multicolumn{3}{|c|}{$p=4$} \\
\hline & LM-Stat & Prob & & LM-Stat & Prob & & LM-Stat & Prob & & LM-Stat & Prob & & LM-Stat & Prob \\
\hline 1 & 52,112 & 0,040 & 1 & 48,202 & 0,084 & 1 & 50,757 & 0,052 & 1 & 43,370 & 0,186 & 1 & 24,554 & 0,926 \\
\hline 2 & 48,150 & 0,085 & 2 & 47,431 & 0,096 & 2 & 40,581 & 0,276 & 2 & 42,090 & 0,224 & 2 & 44,367 & 0,160 \\
\hline 3 & 38,428 & 0,360 & 3 & 42,861 & 0,201 & 3 & 35,402 & 0,497 & 3 & 30,163 & 0,742 & 3 & 35,974 & 0,470 \\
\hline 4 & 48,355 & 0,082 & 4 & 54,530 & 0,025 & 4 & 41,155 & 0,255 & 4 & 50,012 & 0,060 & 4 & 31,247 & 0,694 \\
\hline 5 & 44,261 & 0,162 & 5 & 29,378 & 0,775 & 5 & 27,715 & 0,837 & 5 & 34,924 & 0,520 & 5 & 24,136 & 0,934 \\
\hline 6 & 29,994 & 0,749 & 6 & 43,601 & 0,180 & 6 & 34,302 & 0,550 & 6 & 54,814 & 0,023 & 6 & 52,975 & 0,034 \\
\hline 7 & 32,540 & 0,634 & 7 & 31,064 & 0,702 & 7 & 32,086 & 0,655 & 7 & 38,020 & 0,378 & 7 & 34,209 & 0,554 \\
\hline 8 & 45,978 & 0,123 & 8 & 35,211 & 0,506 & 8 & 44,263 & 0,162 & 8 & 136,231 & 0,000 & 8 & 131,299 & 0,000 \\
\hline 9 & 36,484 & 0,446 & 9 & 60,370 & 0,007 & 9 & 28,810 & 0,797 & 9 & 54,357 & 0,025 & 9 & 30,415 & 0,731 \\
\hline 10 & 32,725 & 0,625 & 10 & 34,424 & 0,544 & 10 & 27,406 & 0,848 & 10 & 42,407 & 0,214 & 10 & 32,327 & 0,644 \\
\hline 11 & 41,202 & 0,254 & 11 & 31,015 & 0,705 & 11 & 39,763 & 0,306 & 11 & 60,520 & 0,006 & 11 & 61,732 & 0,005 \\
\hline 12 & 149,910 & 0,000 & 12 & 89,904 & 0,000 & 12 & 108,228 & 0,000 & 12 & 139,138 & 0,000 & 12 & 88,389 & 0,000 \\
\hline$n$ & 243 & & $n$ & 18 & & $n$ & 243 & & $n$ & 245 & & $\mathrm{n}$ & 24 & \\
\hline
\end{tabular}

Fonte: elaboração própria. Nota: $\mathrm{p}=$ número de defasagens; $\mathrm{n}=$ número de observações incluídas no VAR após definição do número necessário de defasagens.

Tabela 4 - Causalidade de Granger/Teste de Wald de Exogeneidade em Bloco

\begin{tabular}{c|c|c|c|c|c|c|c|c}
\hline \multicolumn{4}{c|}{ Modelo 1} & \multicolumn{3}{c|}{ Modelo 2} & \multicolumn{3}{c}{ Modelo 3 } \\
\hline Variável & $\chi^{2}$ & Prob & Variável & $\chi^{2}$ & Prob & Variável & $\chi^{2}$ & Prob \\
\hline CYCGOV & 4,923 & 0,896 & CYCGOV & 6,720 & 0,752 & CYCGOV & 18,068 & 0,583 \\
\hline CYCCAMBIO & 9,905 & 0,449 & CYCSOLOW & 13,724 & 0,186 & CYCCAMBIO & 26,309 & 0,156 \\
\hline FATOR & 22,202 & 0,014 & FATOR & 26,997 & 0,003 & CYCIND & 30,765 & 0,058 \\
\hline CYCSOLOW & 27,642 & 0,002 & CYCIPCA & 27,928 & 0,002 & CYCSOLOW & 34,838 & 0,021 \\
\hline CYCIPCA & 46,356 & 0,000 & CYCIBC & 34,760 & 0,000 & FATOR & 33,836 & 0,027 \\
\hline CYCPIB & 71,521 & 0,000 & CYCCAMBIO & 40,817 & 0,000 & CYCIPCA & 66,786 & 0,000 \\
\hline
\end{tabular}

Modelo 4 Modelo 5

\begin{tabular}{c|c|cc|c|c}
\hline Variável & $\chi^{2}$ & Prob & Variável & $\chi^{2}$ & Prob \\
\hline CYCGOV & 5,404 & 0,863 & CYCGOV & 14,652 & 0,796 \\
\hline HAMCAMBIO & 7,232 & 0,703 & FATOR & 15,948 & 0,720 \\
\hline FATOR & 15,020 & 0,131 & HAMCAMBIO & 18,080 & 0,582 \\
\hline HAMSOLOW & 16,203 & 0,094 & HAMIND & 24,233 & 0,232 \\
\hline HAMIPCA & 39,485 & 0,000 & HAMSOLOW & 31,799 & 0,046 \\
\hline HAMPIB & 58,910 & 0,000 & HAMIPCA & 69,421 & 0,000 \\
\hline
\end{tabular}

Fonte: elaboração própria. 
A segunda estratégia estimou funções de resposta a impulsos generalizados propostas por Pesaran e Shin (1998). Diversamente das funções de resposta a impulsos com decomposição de Cholesky, as funções de resposta a impulsos generalizados apresentam resultados que independem do ordenamento das variáveis. Para os referidos autores, os resultados obtidos com essas funções são únicos e consideram os padrões históricos de correlação que são observados entre os diversos choques.

Pesaran e Shin (1998) descrevem a função de resposta a impulsos generalizados como o perfil temporal do efeito de um hipotético vetor $\mathrm{m} \times 1$ de choques de tamanho $\delta=\left(\delta_{1}, \ldots, \delta_{m}\right)$. Definindo os dados econômicos conhecidos até o período $t-1$ pelo conjunto de informações não decrescentes $\Omega_{t-1}$, a função de resposta a impulso generalizada de $x_{t}$ no horizonte n é definida como:

$$
G I_{x}\left(n, \delta, \Omega_{t-1}\right)=E\left(\mathrm{x}_{t+n} \mid \varepsilon_{t}=\delta, \Omega_{t-1}\right)-E\left(\mathrm{x}_{t+n} \mid \Omega_{t-1}\right),
$$

em que $G I_{x}\left(n, \delta, \Omega_{t-1}\right)=A_{n} \delta$, o qual é independente de $\Omega_{t-1}$. A função depende da composição de choques definidos por $\delta$; ou seja, o vetor de choques é sua propriedade central. A próxima seção apresenta a análise das funções de resposta a impulsos e das decomposições de variâncias dos modelos estimados.

\section{Resultados}

A análise dos modelos VAR foi realizada para o período entre janeiro de 1998 e julho de 2017, em razão de restrições na disponibilidade de dados para CYCGOV. Nos modelos estimados com o componente cíclico do IBC, a indisponibilidade de dados restringiu a amostra para o período entre janeiro de 2003 a julho de 2018.

A Figura 2 apresenta os resultados das funções de resposta a impulsos generalizados. Como os resultados obtidos com a ordenação de Cholesky mostraram-se idênticos, foram omitidos. ${ }^{10}$ Inicialmente, ilustram-se apenas os resultados de choques em FATOR e nos componentes cíclicos do

${ }^{10}$ Esses resultados podem ser obtidos mediante solicitação aos autores. 
produto e produção industrial. O comportamento das demais variáveis é discutido adiante.

Choques exógenos no indicador de fragilidade financeira levam a uma contração na produção nos primeiros quatro a nove meses subsequentes ao choque. Esse resultado somente não é verificado no modelo (1), quando a volatilidade na produção é medida por CYCPIB. Nos demais modelos, a contração na produção resultante de elevações na fragilidade financeira é mais robusta. Também na Figura 2, é possível constatar que o reverso não procede, ou seja, choques exógenos na produção não afetam a fragilidade financeira.

Os resultados da Figura 2 estão alinhados com os modelos teóricos que enfatizam o impacto macroeconômico de assimetrias de informação no mercado de crédito (Stiglitz 1987, Bernanke et al. 1996, Mishkin 1998, 2000). Uma maior fragilidade financeira é associada com elevações no prêmio de risco e com o racionamento de crédito, o que compromete o desempenho econômico. No entanto, a ausência de resposta da fragilidade financeira a choques positivos na produção é um resultado inesperado na visão do canal do risco (Ryoo 2010, Borio e Zhu 2012, Azariadis 2018). A análise do canal do risco indica que a estabilidade macroeconômica levaria os agentes econômicos a adotarem comportamentos mais arriscados, eventualmente elevando a fragilidade financeira.

Dentre as variáveis de controle, a volatilidade cambial e a inflação são as únicas que afetam de maneira estatisticamente significante o desempenho macroeconômico de curto prazo no Brasil (Figura 3). Funções de resposta a impulsos generalizados mostram que, independente do modelo estimado, choques positivos na volatilidade cambial tem um impacto imediato e prologado sobre a produção, que declina por mais de doze meses após o choque. Já o impacto da elevação da inflação prolonga-se por no máximo seis meses e apresenta um comportamento defasado quando CYCIBC é utilizado como medida de atividade econômica. 

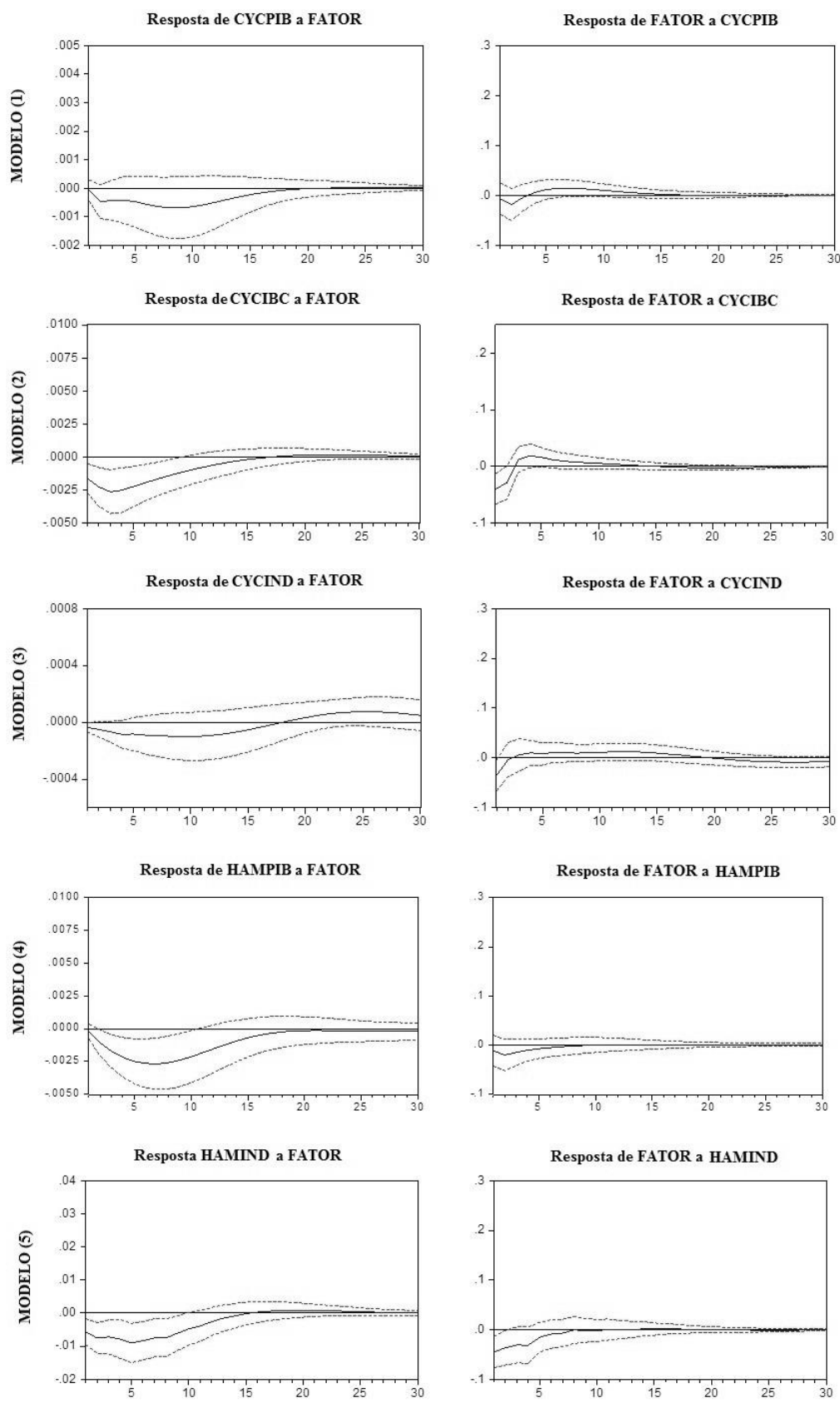

Figura 2 - Inovações na Fragilidade Financeira e na Volatilidade Macroeconômica Fonte: elaboração própria. Nota: respostas a um desvio padrão generalizado ( \pm 2 erros padrão). 

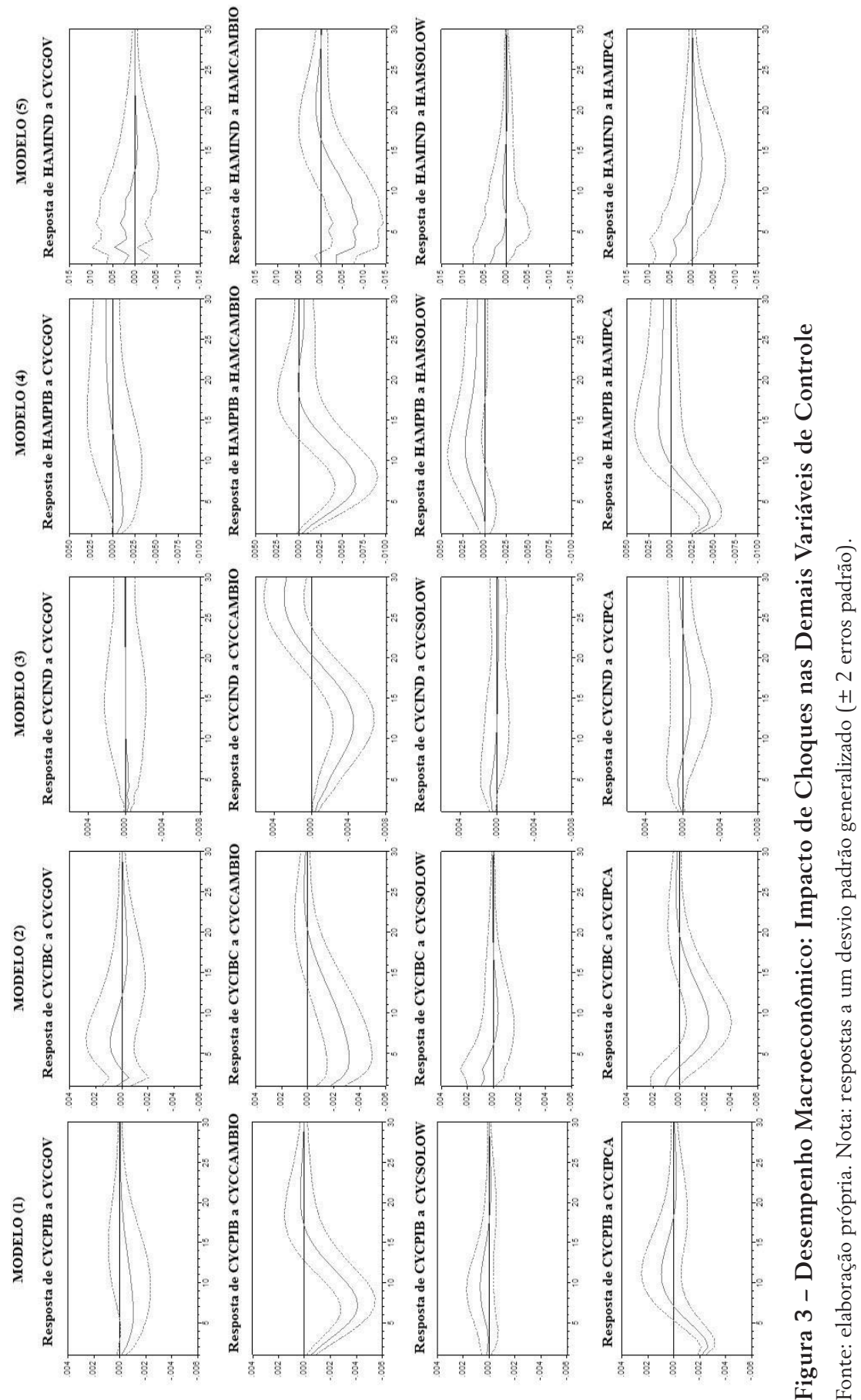
A análise de decomposição de variância por Cholesky ratifica os resultados apresentados anteriormente (Tabela 5). A variância dos indicadores de volatilidade macroeconômica é principalmente explicada pelas suas próprias defasagens e pela volatilidade cambial. Em alguns modelos, o componente cíclico da inflação também afeta a volatilidade macroeconômica. Quando CYCIBC, HAMIND e, em menor grau, HAMPIB representam o componente cíclico da produção, a fragilidade financeira apresenta um papel de maior destaque para explicar a volatilidade macroeconômica.

Choques na volatilidade cambial são os principais determinantes da variância das demais variáveis endógenas dos modelos estimados, com exceção do resíduo de Solow e dos gastos governamentais, que são explicados principalmente pelas suas próprias defasagens. Utilizando os resultados do Modelo 1 como ilustração, observa-se que, doze meses após o choque, cerca de $62 \%$ da variância no PIB e $50 \%$ da variância do IPCA podem ser atribuídos à maior volatilidade cambial (ver Apêndice 2). Já a variância do indicador de fragilidade financeira é explicada, principalmente, pelas suas próprias defasagens e pelo componente cíclico da inflação.

Como teste final de robustez, os modelos foram estimados sem incluir CYCGOV e CYCSOLOW, uma vez que essas variáveis não demostraram ter impacto relevante sobre o desempenho da produção. A exclusão de CYCGOV, em particular, permite estimar os modelos com amostras mais longas. ${ }^{11}$ Observa-se que os resultados apresentados na Figura 4 são semelhantes àqueles ilustrados na Figura 2: choques que elevam a fragilidade financeira levam ao declínio da produção durante os primeiros cinco meses subsequentes ao choque na maior parte dos modelos, exceto no modelo (5), quando a resposta negativa da produção perdura por dez meses. Verifica-se, também, que choques exógenos na produção produzem uma leve redução da fragilidade financeira nos modelos (1) e (2).

${ }^{11}$ O Modelo 1 foi estimado com 265 observações, enquanto os modelos 2, 3, 4 e 5 foram estimados com 184, 261, 251 e 244, respectivamente. 


\section{Conclusões}

Este trabalho se propôs a analisar a relação entre fragilidade financeira e o desempenho macroeconômico de curto prazo da economia brasileira. Para isso, foram selecionadas variáveis diversas que refletem a fragilidade financeira, tais como prêmios de risco, robustez do sistema financeiro e medidas de expansão do crédito. Utilizou-se um modelo de fator dinâmico para derivar o índice de fragilidade financeira e estimou-se a relação entre este indicador e os componentes cíclicos de variáveis macroeconômicas com o uso de modelos de vetores autorregressivos.

Tabela 5 - Decomposição da Variância dos Componentes Cíclicos da Produção em \%

\begin{tabular}{|c|c|c|c|c|c|c|c|}
\hline \multicolumn{8}{|c|}{ Modelo 1 - CYCPIB } \\
\hline Mês & S.E. & FATOR & CYCGOV & CYCCAMBIO & CYCSOLOW & CYCIPCA & CYCPIB \\
\hline 1 & 0,003 & 0,001 & 0,217 & 3,595 & 0,496 & 60,327 & 35,364 \\
\hline 6 & 0,009 & 0,117 & 4,299 & 47,415 & 0,667 & 22,833 & 24,669 \\
\hline 12 & 0,013 & 0,104 & 4,805 & 62,410 & 2,127 & 14,681 & 15,873 \\
\hline 18 & 0,014 & 0,148 & 5,262 & 61,819 & 2,273 & 15,104 & 15,393 \\
\hline 24 & 0,014 & 0,154 & 5,284 & 61,689 & 2,314 & 15,133 & 15,426 \\
\hline \multicolumn{8}{|c|}{ Modelo 2 - CYCIBC } \\
\hline Mês & S.E. & FATOR & CYCGOV & CYCCAMBIO & CYCSOLOW & CYCIPCA & CYCIBC \\
\hline 1 & 0,002 & 3,984 & 0,243 & 0,000 & 1,586 & 1,427 & 92,760 \\
\hline 6 & 0,003 & 13,764 & 1,002 & 7,350 & 1,186 & 2,998 & 73,700 \\
\hline 12 & 0,004 & 13,874 & 1,446 & 13,445 & 1,095 & 12,167 & 57,973 \\
\hline 18 & 0,004 & 13,287 & 1,612 & 14,924 & 1,080 & 13,417 & 55,680 \\
\hline 24 & 0,004 & 13,255 & 1,814 & 14,887 & 1,077 & 13,379 & 55,587 \\
\hline \multicolumn{8}{|c|}{ Modelo 3 - CYCIND } \\
\hline Mês & S.E. & FATOR & CYCGOV & CYCCAMBIO & CYCSOLOW & CYCIPCA & CYCIND \\
\hline 1 & 0,002 & 0,000 & 0,470 & 2,858 & 0,000 & 0,000 & 96,673 \\
\hline 6 & 0,003 & 0,007 & 0,449 & 12,999 & 0,360 & 0,545 & 85,640 \\
\hline 12 & 0,003 & 0,003 & 0,217 & 38,406 & 0,644 & 0,342 & 60,388 \\
\hline 18 & 0,004 & 0,013 & 0,168 & 50,129 & 0,554 & 0,651 & 48,486 \\
\hline 24 & 0,004 & 0,052 & 0,154 & 47,635 & 0,655 & 0,644 & 50,861 \\
\hline \multicolumn{8}{|c|}{ Modelo 4 - HAMPIB } \\
\hline Mês & S.E. & FATOR & CYCGOV & HAMCAMBIO & HAMSOLOW & HAMIPCA & HAMPIB \\
\hline 1 & 0,002 & 0,121 & 0,962 & 0,737 & 0,006 & 39,916 & 58,259 \\
\hline 6 & 0,003 & 4,081 & 1,938 & 31,282 & 0,481 & 27,745 & 34,474 \\
\hline 12 & 0,003 & 4,359 & 1,354 & 45,899 & 4,365 & 14,629 & 29,394 \\
\hline 18 & 0,003 & 4,137 & 1,295 & 42,436 & 6,859 & 13,722 & 31,551 \\
\hline 24 & 0,003 & 3,969 & 1,678 & 40,497 & 7,578 & 13,546 & 32,732 \\
\hline \multicolumn{8}{|c|}{ Modelo 5 - HAMIND } \\
\hline Mês & S.E. & FATOR & CYCGOV & HAMCAMBIO & HAMSOLOW & HAMIPCA & HAMIND \\
\hline 1 & 0,002 & 3,310 & 0,657 & 0,994 & 0,000 & 0,000 & 95,038 \\
\hline 6 & 0,003 & 14,420 & 2,039 & 8,937 & 0,384 & 0,596 & 73,624 \\
\hline 12 & 0,004 & 18,584 & 2,249 & 14,323 & 0,461 & 0,725 & 63,659 \\
\hline 18 & 0,004 & 18,382 & 2,248 & 14,516 & 0,490 & 1,436 & 62,929 \\
\hline 24 & 0,004 & 18,399 & 2,243 & 14,599 & 0,492 & 1,619 & 62,648 \\
\hline
\end{tabular}

Fonte: elaboração própria. Nota: decomposição por Cholesky com ordenamento conforme indicado na Tabela 4; S.E. = erro padrão. 

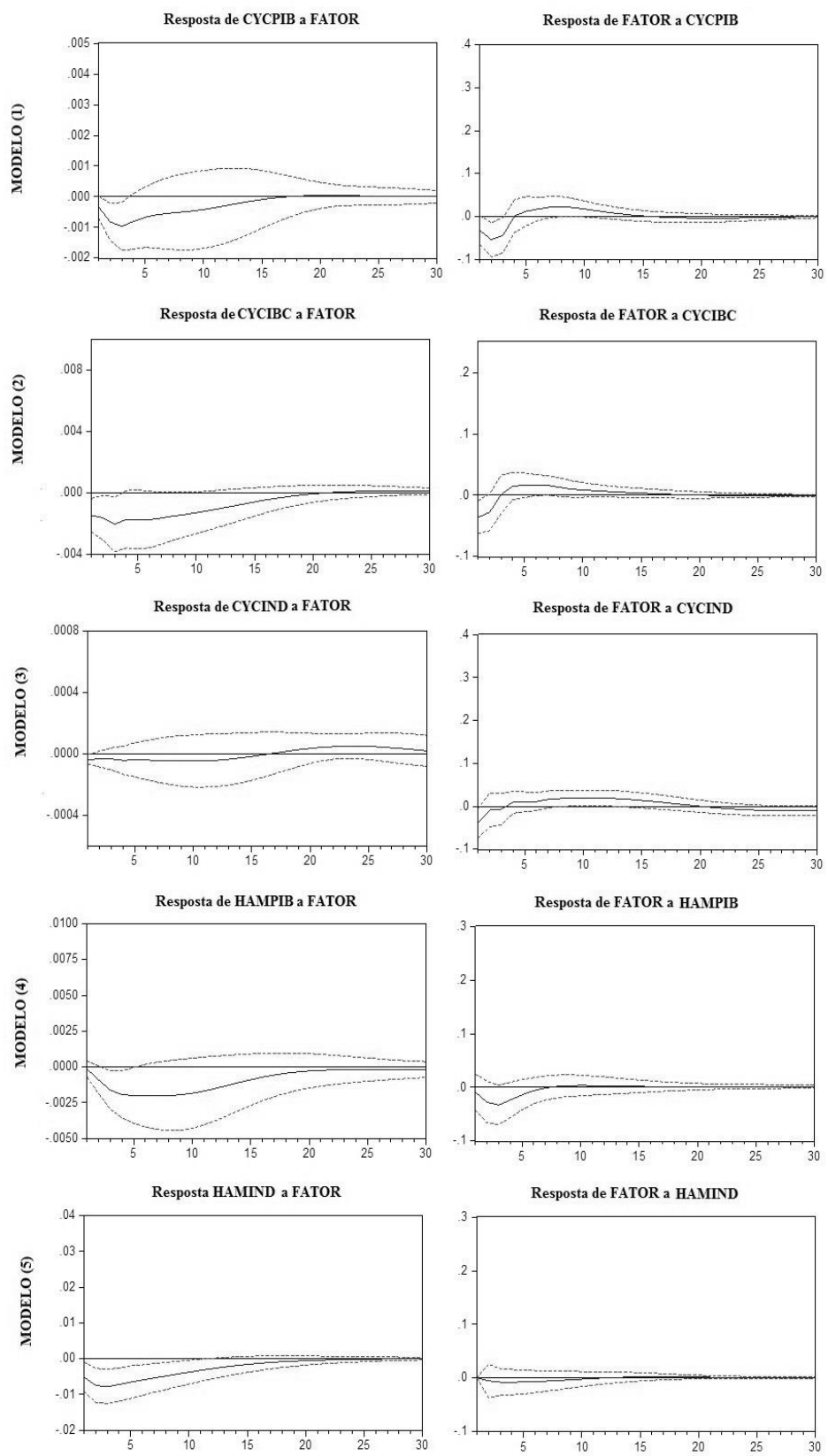

Figura 4 - Inovações na Fragilidade Financeira e na Volatilidade Macroeconômica

Fonte: elaboração própria. Nota: respostas a um desvio padrão generalizado ( \pm 2 erros padrão). Variáveis endógenas incluídas nas estimações: CYCCAMBIO e CYCIPCA. 
Os resultados mostram que choques exógenos na fragilidade financeira levam a contrações na atividade econômica no Brasil, indicando que a deterioração nas condições para a concessão de crédito e no funcionamento do mercado financeiro contribui para a contração na atividade econômica. Embora choques na variável de fragilidade financeira apresentem um efeito estatisticamente significante sobre o produto agregado, choques nos componentes cíclicos do produto interno bruto, do IBC-BR e da produção industrial parecem não afetar de maneira significativa o índice de fragilidade financeira.

Este resultado indica que uma maior fragilização do sistema financeiro, ilustrada pela elevação nos prêmios de risco e por um crescente racionamento de crédito, provoca uma contração na atividade econômica. Esta evidência corrobora com análises teóricas de tradição Keynesiana que enfatizam a importância de um sistema financeiro robusto para a estabilidade macroeconômica.

No entanto, os resultados também mostram que expansões na atividade econômica não produzem maior fragilidade financeira, nem no curto, nem no longo prazo. Este resultado é mais difícil de conciliar com a visão de que os agentes econômicos adotam comportamentos mais arriscados quando o otimismo é prevalente (e.g. Ryoo 2010, Borio e Zhu 2012, Azariadis 2018).

Por fim, os resultados também mostram a importância de choques cambiais e de choques no nível de preços para o desemprenho macroeconômico de curto prazo. Aumentos na volatilidade cambial, em particular, contribuem consistentemente para inibir a atividade econômica e na elevação do nível de preços. 


\section{Referências}

Aghion, P., Angeletos, M., Banerjee, A., E Manova, K. "Volatility and growth: Credit constraints and the composition of investment." Journal of Monetary Economics 57, no. 3: 246-65, 2010.

Alesina, A., Campante, F.R., e Tabellini, G. "Why is fiscal policy often procyclical?" Journal of the European Economic Association 6: 1006-36, 2008.

Aruoba, S. B.; Diebold, F.X.; Scotti, C. "Real-time measurement of business conditions." Journal of Business \& Economic Statistics 27, no. 4: 417-427, 2009.

Azariadis, C. "Credit Cycles and Business Cycles." Federal Reserve Bank of St. Louis Review 100, no.1: 45-71, 2018.

Baker, S.R.; Bloom, N.; Davis, S.J. "Measuring economic policy uncertainty." The quarterly journal of economics 131, no.4: 1593-1636, 2016.

BANCO CENTRAL DO BRASIL (BCB). "Séries Temporais (SGS)". Brasília, 2019. Disponível em: https:// www.bcb.gov.br>. Acesso em 28 fev. 2019.

Backus, D., Kehoe, P., e Kydland, F. "International real business cycles." Journal of Political Economy 100: 745-775, 1992.

Baxter, M.; King, R.G. "Measuring business cycles: approximate band-pass filters for economic time series." Review of economics and statistics 81, no.4: 575-593, 1999.

Beck, T., Demirguç-Kunt, A., E Levine, R. "Finance, Inequality and the Poor." Journal of Economic Growth 12, no.1: 27-49, 2007.

Beck, T., Chen, T., Lin, C., E Song, F. M. "Financial innovation: The bright and the dark sides." Journal of Banking \& Finance 72: 28-51, 2016.

Bernanke, B.S., Gertler, M., e Gilchrist, S. "The financial accelerator and the flight to quality." Review of Economics and Statistics 78: 1- 15, 1996.

Bernanke, B.S., Gertler, M., e Gilchrist, S. "The financial accelerator in a quantitative business cycle framework." In: Taylor, J., Woodford, M. Handbook of Macroeconomics 1B: 1342-1393, 1999.

Bernanke, B.S., e Lown, C. “The credit crunch.” Brookings Papers on Economic Activity 2: 205-247, 1991.

Borio, C., e Zhu, H. "Capital regulation, risk-taking and monetary policy: a missing link in the transmission mechanism?" Journal of Financial Stability 8, no. 4: 236-51, 2012.

Caballero, R. J., e Krishnamurthy, A. "International and Domestic Collateral Constraints in a Model of Emerging Market Crises." Journal of Monetary Economics 48: 513-548, 2001.

Chang, Y.S., e Pak, D. "Warren Buffett value indicator vs. GDP size-is the relationship superlinear?" International Journal of Economics and Business Research 15, no.2: 223-235, 2018.

Charles, A., Darné, O., e Tripier, F. "Uncertainty and the Macroeconomy: Evidence from an uncertainty composite indicator." Applied Economics 50, no.10: 1093-107, 2018.

Christiano, L.J., e Fitzgerald, T.J. “The band pass filter.” International Economic Review 44, no.2: 435-465, 2003.

Christiano, L.J.; Eichenbaum, M.; Evans, C.L. "Nominal rigidities and the dynamic effects of a shock to monetary policy." Journal of Political Economy 113, no.1: 1-45, 2005.

Čihák, M., Demirgüč-Kunt, A., Feyen, E. e Levine, R. "Financial development in 205 economies, 1960 to 2010.” NBER Working Paper 18943, 2013.

COMITÊ DE DATAÇÃO DOS CICLOS ECONÔMICOS (CODACE). 2017. Comunicado de Datação de Ciclos Mensais Brasileiros - Out/2017. Rio de Janeiro: IBRE.

Dabla-Norris, E., e Srivisal, N. "Revisiting the Link between Finance and Macroeconomic Volatility." United Kingdom, 2013. IMF Working Paper 29. 
Denizer, C.A., Iyigun, M.F., e Owen, A. "Finance and Macroeconomic Volatility." The B.E. Journal of Macroeconomics 2, no.1: 1-32, 2002.

Easterly, W., Islam, R. e Stiglitz, J.E. "Shaken and stirred: explaining growth volatility". In: Annual World Bank conference on development economics. World Bank, 191-211, 2001.

Fazzari, S., Ferri, P., e Greenberg, E. “Cash flow, investment, and Keynes-Minsky cycles.” Journal of Economic Behavior \& Organization 65, no.3-4: 555-572, 2008.

Gennaioli, N., Shleifer, A., e Vishny, R. "Neglected risks, financial innovation, and financial fragility." Journal of Financial Economics 104, no.3: 452-468, 2012.

Gertler, M. "Financial structure and aggregate economic activity: an overview." Journal of Money, Credit and Banking 20:559-588, 1988.

Geweke, J. "The dynamic factor analysis of economic time series models". In: Latent Variables in Socioeconomic Models, ed. D. J. Aigner and A. S. Goldberger. (Amsterdam: North-Holland, 1977): 365-383.

Gilchrist, S., SIM, J. W., e Zakrajsek, E. "Uncertainty, Financial Frictions, and Investment Dynamics.” NBER Working Paper, 20038, 2014.

Gilchrist, S., e Zakrajsek, E. "Credit Spreads and Business Cycle Fluctuations." American Economic Review 102: 1692-1720, 2012.

Hamilton, J.D. "Why you should never use the Hodrick-Prescott filter." Review of Economics and Statistics 100(5): 831-843, 2018.

INSTITUTO BRASILEIRO DE GEOGRAFIA E ESTATÍSTICA (IBGE). 2017. PEA. Disponível em: < http:// seriesestatisticas.ibge.gov.br/>. Acesso em: 15 ago. 2017.

Jurado, K., Ludvigson, S.C., e NG, S. "Measuring uncertainty.” American Economic Review 105(3): 1177-1216, 2015.

Karras, G., e Song, F. "Sources of business-cycle volatility: an exploratory study on a sample of OECD countries." Journal of Macroeconomics 18: 621-637, 1996.

King, R., e Levine, R. "Finance and growth: Schumpeter might be right." Quarterly Journal of Economics 108: 717-737, 1993.

Kliesen, K.L., Owyang, M.T. e Vermann, E.K. "Disentangling diverse measures: A survey of financial stress indexes." Federal Reserve Bank of St. Louis Review 94, no.5: 369-397, 2012.

Kydland, F.E.; Prescott, E.C. "Time to build and aggregate fluctuations." Econometrica: Journal of the Econometric Society, 1345-1370, 1982.

Levine, R. "Finance and Growth: Theory and Evidence". In: Aghion, P., Durlauf, S. Handbook of Economic Growth 1: 865-934, 2005, 1. ed.

Loayza, N., e Raddatz, C. "The Structural Determinants of External Vulnerability." The World Bank Economic Review 21: 359-387, 2007.

Long, Jr., J.B., e Plosser, C.I. “Real business cycles.” Journal of Political Economy 91, no.1: 39-69, 1983.

Lütkepohl, H. "New introduction to multiple time series analysis.” Berlim: Springer Science \& Business Media, 2005 .

Marques-Ibanez, D., Altunbas, Y., e Van Leuvensteijn, M. "Competition and bank risk: the effect of securitization and bank capital.” ECB Working Paper, n. 1678, 2014.

Minsky, H. P. “Stabilizing an Unstable Economy.” Yale University Press, 1986.

Mishkin, F.S. "International Capital Movements, Financial Volatility and Financial Instability.” NBER Working Paper. n. 6390, 1998.

Mishkin, F.S. "Financial Stability and the Macroeconomy." Iceland: Department of Economics. Central Bank of Iceland Working Paper 9, 2000. 
Pesaran, H.H, e Shin, Y. "Generalized impulse response analysis in linear multivariate models." Economics Letters 58: 17-29, 1998.

Poncela, P., e Ruiz, E. "Small-versus big-data factor extraction in dynamic factor models: An empirical assessment”. In: Dynamic Factor Models. Emerald Group Publishing Limited, 401-434, 2016.

Popov, A. "Financial Liberalization, Growth, and Risk.” European Central Bank, manuscrito, 2011.

Pysarenko, S., Alexeev, V., e Tapon, F. "Predictive blends: Fundamental Indexing meets Markowitz." Journal of Banking \& Finance 100: 28-42, 2019.

Quah, D., e Sargent, T.J. “A dynamic index model for large cross sections. In: Business cycles, indicators and forecasting." University of Chicago Press, 1993: 285-310.

Ramey, G., e Ramey, V. "Cross-country evidence on the link between volatility and growth." NBER Working Paper 4959, 1994.

Rebelo, S.T. "Real Business Cycle Models: Past, Present and Future." Scandinavian Journal of Economics 107, no.2: 217-238, 2005.

Ryoo, S. "Long waves and short cycles in a model of endogenous financial fragility." Journal of Economic Behavior \& Organization 74, no.3: 163-186, 2010.

Schüler, Y.S. "On the cyclical properties of Hamilton's regression filter.” Deutsche Bundesbank Discussion Paper 3, 2018.

Silva, G. F. "The impact of financial system development on business cycles volatility: cross-country evidence." Journal of Macroeconomics 24: 233-253, 2002.

Smets, F., e Wouters, R. "Shocks and frictions in US business cycles: A Bayesian DSGE approach." American Economic Review 97, no. 3: 586-606, 2007.

Stiglitz, J.E. "The Causes and Consequences of the Dependence of Quality on Price." Journal of Economic Literature 25, no.1, 1987.

Stock, J.H., e Watson, M. "Dynamic factor models." Oxford Handbook on Economic Forecasting, 2011.

TESOURO NACIONAL. 2019. SIAFI. Disponível em: < http://www.tesouro.fazenda.gov.br/-/series-historicas>. Acesso em 15 fev. 2019.

Teixeira, D. N.; Tiryaki, G.F. "Fragilidade financeira e ciclos econômicos no Brasil." Revista Desenbahia 14, no. 23: 165-200, 2017.

Tiryaki, G.; Gavazza, I.; Mota, A.; Malbouisson, C. "Ciclos de Crédito, Inadimplência e as Flutuações Econômicas no Brasil.” Revista de Economia Contemporânea 21, no.1: 1-33, 2017.

WORLD BANK. 2017. Global Financial Development Database. Washington: World Bank. Disponível em: http://databank.worldbank.org/data/reports.aspx?source=global-financial-development\#. Acesso em 07 nov. 2017. 


\section{APÊNDICE 1 - TESTES DE RAIZ UNITÁRIA}

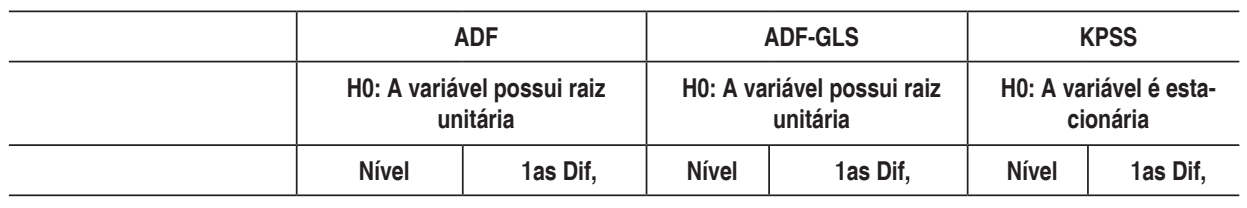

VARIÁVEL: BOVPIB

\begin{tabular}{|c|c|c|c|c|c|c|}
\hline Constante & $-1,873$ & $-15,977^{\star \star \star}$ & $-0,728$ & $-14,839^{\star * *}$ & 1,074 & 0,084 \\
\hline Constante e Tendência & $-1,896$ & $-15,968^{* \star *}$ & $-1,796$ & $-15,534^{\star \star *}$ & 0,352 & 0,045 \\
\hline \multicolumn{7}{|l|}{ VARIÁVEL: CHQSELIC } \\
\hline Constante & $-1,397$ & $-2,877^{\star *}$ & $-1,612$ & $-2,582^{\star \star *}$ & 1,067 & 0,548 \\
\hline Constante e Tendência & $-2,112$ & $-2,846$ & $-1,747$ & $-2,949^{\star *}$ & 0,368 & 0,063 \\
\hline \multicolumn{7}{|l|}{ VARIÁVEL: PJSELIC } \\
\hline Constante & $-3,422^{\star \star}$ & - & 0,437 & $-0,631$ & 1,561 & 0,198 \\
\hline Constante e Tendência & $-4,125^{\star \star \star}$ & - & $-1,359$ & $-1,631$ & 0,305 & 0,078 \\
\hline \multicolumn{7}{|l|}{ VARIÁVEL: PFSELIC } \\
\hline Constante & $-4,658^{* * *}$ & - & 0,785 & $-0,292$ & 1,808 & 0,474 \\
\hline Constante e Tendência & $-4,193^{* \star *}$ & - & $-0,408$ & $-1,405$ & 0,374 & 0,071 \\
\hline \multicolumn{7}{|l|}{ VARIÁVEL: PROVPRIV } \\
\hline Constante & $-5,931^{* * *}$ & - & $-0,464$ & $-2,136^{\star *}$ & 0,221 & - \\
\hline Constante e Tendência & $-6,101^{* \star *}$ & - & $-1,625$ & $-4,077^{\star * *}$ & 0,221 & 0,065 \\
\hline \multicolumn{7}{|l|}{ VARIÁVEL: PROVPUB } \\
\hline Constante & $-1,551$ & $-17,049^{\star * *}$ & $-1,557$ & $-5,990^{\star * \star}$ & 1,608 & 0,079 \\
\hline Constante e Tendência & $-2,982$ & $-17,021^{* * *}$ & $-1,882$ & $-16,836^{\star \star *}$ & 0,179 & - \\
\hline \multicolumn{7}{|l|}{ VARIÁVEL: M1PIB } \\
\hline Constante & $-3,310^{\star *}$ & - & 0,029 & $-2,571^{\star \star}$ & 0,922 & 0,585 \\
\hline Constante e Tendência & $-2,233$ & $-17,875^{\star \star \star}$ & $-0,407$ & $-3,730^{\star \star *}$ & 0,496 & 0,048 \\
\hline \multicolumn{7}{|l|}{ VARIÁVEL: CREDTOT } \\
\hline Constante & $-2,425$ & $-16,706^{* * *}$ & $-0,050$ & $-6,720^{\star * *}$ & 0,688 & - \\
\hline Constante e Tendência & $-1,609$ & $-17,040^{\star \star *}$ & $-0,469$ & $-15,680^{\star * *}$ & 0,446 & 0,091 \\
\hline \multicolumn{7}{|l|}{ VARIÁVEL: PRIVY } \\
\hline Constante & $-0,210$ & $-14,793^{* * *}$ & 0,519 & $-13,130^{\star * *}$ & 1,878 & 0,467 \\
\hline Constante e Tendência & $-1,417$ & $-14,777^{* * *}$ & $-0,665$ & $-14,408^{\star * *}$ & 0,298 & 0,438 \\
\hline
\end{tabular}

Fonte: elaboração própria. Notas: os valores na tabela representam a estatística do teste realizado. O número máximo de defasagens ( $\mathrm{p}_{\operatorname{MAX}}$ ) utilizado foi 15 , com base no critério de Schawarz e de acordo com a expressão $\mathrm{p}_{\mathrm{MAX}}=\left[12(\mathrm{~T} / 100)^{(1 / 4)}\right]$, onde T é o número de observações (Schwert 1989). Significância estatística de $1 \%, 5 \%$ e $10 \%$ são indicados por ${ }^{* * *},{ }^{* *} \mathrm{e}^{*}$, respectivamente. 


\section{APÊNDICE 1 - DECOMPOSIÇÃO DE VARIÂNCIA: MODELO 1}

\begin{tabular}{|c|c|c|c|c|c|c|c|}
\hline \multicolumn{8}{|c|}{ FATOR } \\
\hline Mês & S.E. & FATOR & CYCGOV & CYCCAMBIO & CYCSOLOW & CYCIPCA & CYCPIB \\
\hline 1 & 0,017 & 0,024 & 0,538 & 5,685 & 0,352 & 57,197 & 36,204 \\
\hline 6 & 0,080 & 34,831 & 2,865 & 3,645 & 2,339 & 28,453 & 27,867 \\
\hline 12 & 0,088 & 62,262 & 4,194 & 2,121 & 1,659 & 15,795 & 13,969 \\
\hline 18 & 0,092 & 60,551 & 3,947 & 2,282 & 4,453 & 15,848 & 12,919 \\
\hline 24 & 0,093 & 59,766 & 4,103 & 2,293 & 5,641 & 15,634 & 12,563 \\
\hline \multicolumn{8}{|c|}{ CYCGOV } \\
\hline Mês & S.E. & FATOR & CYCGOV & CYCCAMBIO & CYCSOLOW & CYCIPCA & CYCPIB \\
\hline 1 & 0,021 & 0,000 & 100,000 & 0,000 & 0,000 & 0,000 & 0,000 \\
\hline 6 & 0,084 & 0,041 & 96,651 & 0,665 & 0,606 & 0,789 & 1,248 \\
\hline 12 & 0,090 & 0,035 & 93,068 & 0,620 & 1,137 & 3,307 & 1,832 \\
\hline 18 & 0,091 & 0,035 & 91,853 & 0,620 & 1,290 & 4,293 & 1,908 \\
\hline 24 & 0,091 & 0,035 & 91,734 & 0,665 & 1,295 & 4,365 & 1,906 \\
\hline \multicolumn{8}{|c|}{ CYCCAMBIO } \\
\hline Mês & S.E. & FATOR & CYCGOV & CYCСАМВIO & CYCSOLOW & CYCIPCA & CYCPIB \\
\hline 1 & 0,243 & 0,000 & 0,169 & 99,831 & 0,000 & 0,000 & 0,000 \\
\hline 6 & 0,281 & 0,286 & 0,106 & 93,328 & 0,925 & 4,766 & 0,590 \\
\hline 12 & 0,289 & 0,450 & 0,373 & 90,293 & 1,289 & 6,797 & 0,799 \\
\hline 18 & 0,292 & 0,445 & 0,378 & 89,253 & 1,482 & 7,239 & 1,202 \\
\hline 24 & 0,292 & 0,444 & 0,377 & 88,988 & 1,527 & 7,411 & 1,253 \\
\hline \multicolumn{8}{|c|}{ CYCSOLOW } \\
\hline Mês & S.E. & FATOR & CYCGOV & CYCCAMBIO & CYCSOLOW & CYCIPCA & CYCPIB \\
\hline 1 & 0,004 & 0,248 & 0,182 & 0,181 & 99,389 & 0,000 & 0,000 \\
\hline 6 & 0,004 & 0,604 & 0,284 & 0,344 & 89,929 & 4,669 & 4,171 \\
\hline 12 & 0,004 & 0,652 & 0,325 & 0,693 & 88,020 & 5,330 & 4,979 \\
\hline 18 & 0,004 & 0,662 & 0,325 & 0,768 & 87,456 & 5,683 & 5,107 \\
\hline 24 & 0,004 & 0,661 & 0,329 & 0,818 & 87,345 & 5,738 & 5,109 \\
\hline \multicolumn{8}{|c|}{ CYCIPCA } \\
\hline Mês & S.E. & FATOR & CYCGOV & CYCCAMBIO & CYCSOLOW & CYCIPCA & CYCPIB \\
\hline 1 & 0,003 & 0,072 & 0,051 & 2,014 & 4,413 & 93,450 & 0,000 \\
\hline 6 & 0,009 & 0,844 & 0,731 & 35,263 & 5,258 & 56,815 & 1,088 \\
\hline 12 & 0,011 & 1,012 & 2,706 & 49,612 & 3,891 & 41,934 & 0,845 \\
\hline 18 & 0,011 & 1,047 & 3,626 & 49,273 & 3,846 & 41,348 & 0,859 \\
\hline 24 & 0,011 & 1,044 & 3,744 & 49,229 & 3,852 & 41,243 & 0,888 \\
\hline
\end{tabular}

Fonte: elaboração própria. Nota: decomposição por Cholesky com ordenamento conforme indicado na Tabela 4; S.E. = erro padrão. 